\title{
Scent-free policies generally unjustified
}

$\mathrm{T}$ he list of symptoms people with scent sensitivities attribute to chemical fragrances is a lengthy one that includes everything from coughing, sneezing, gagging, shortness of breath, rhinitis and asthma attacks, to debilitating headaches, anxiety and dizziness.

As a result, many workplaces and institutions - schools, hospitals and other government buildings - have some sort of scent-free or scent-reduction policy in place, which asks people entering the building not to wear perfumed products.

But the science supporting such policies is fuzzy and inconclusive. While scents can trigger both physiological and psychological symptoms in some individuals, there is no reliable diagnostic test for fragrance allergies.

Allergies to substances where a protein is easily identifiable can be tested with a skin test, so determining an allergy to peanuts, cat dander or pollen requires only a simple scratch test. Scents, however, are more complicated, as one fragrance can be made up of many different ingredients.

"When someone is smelling something, what they're smelling is usually not the protein, it's the volatile hydrocarbon, or whatever is giving off that scent," says Dr. Susan Waserman, professor of medicine in the division of clinical immunology and allergy at McMaster University in Hamilton, Ontario. As such, scent sensitivities are usually a reaction to an irritant when it reaches a certain concentration, not an allergy to a specific protein.

Much of North American research into scent sensitivities comes from the Monell Chemical Senses Center, an independent, nonprofit scientific institute in Philadelphia, Pennsylvania, where researchers study taste and smell. Pamela Dalton, a psychologist at the center, says that simply telling people that an odourous material is going to cause an adverse reaction is enough to make the test subjects feel poorly, even if the odour is entirely innocuous.

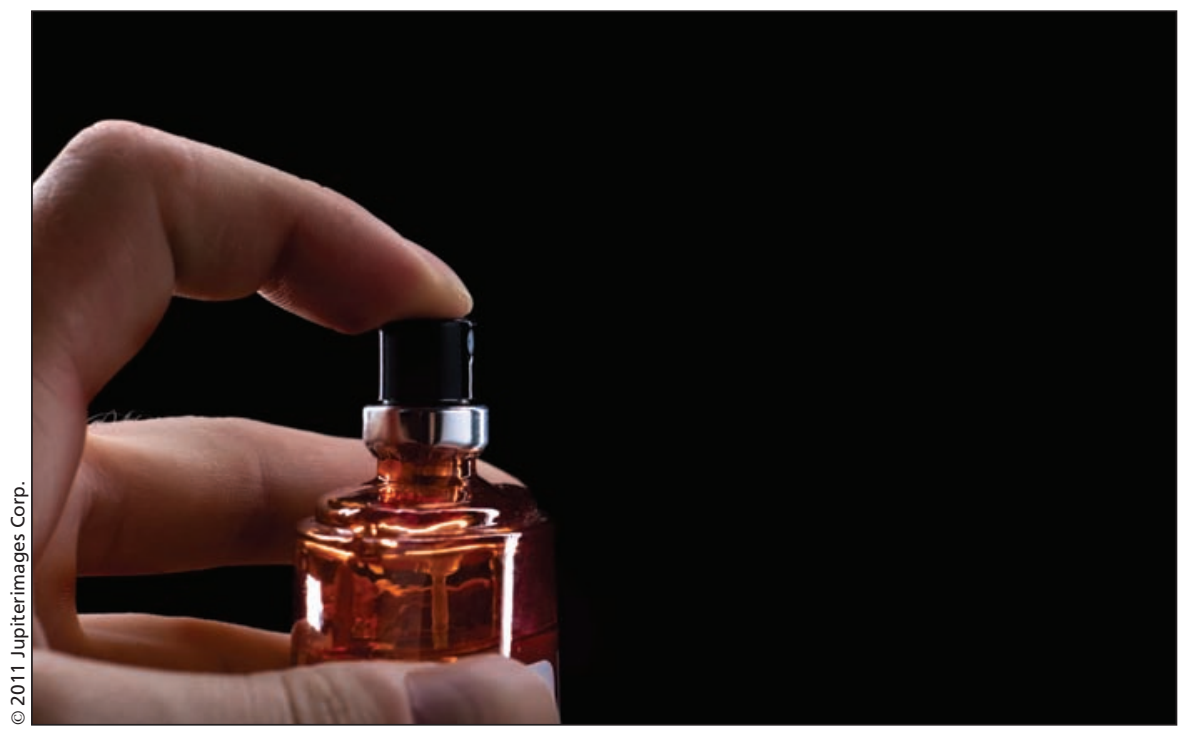

While scents can trigger both physiological and psychological symptoms in some individuals, there is no reliable diagnostic test for fragrance allergies.

People who have one adverse reaction to a scent, or who associate a scent with adverse reactions, can develop anxiety about being exposed to fragrances in future instances, she says.

"When it comes to scents that are used in air fresheners, or someone else's perfume or carpet freshener or that sort of thing, there is an element of loss of control that I think plays in the sense of personal space and that their lungs are being invaded," Dalton adds. "I think that heightens the anxiety about the exposure." This anxiety can cause real physical symptoms, including elevated heart rate and blood pressure, rapid breathing, increased stress hormones and hyperventilation. It can also cause people to isolate themselves for fear of coming into contact with a trigger scent.

Dalton says some fragrance materials have direct physiological effects, but she estimates those affected are probably a smaller subset of individuals than the ones who have a more psychological-based reaction.

Since there is no diagnostic test to confirm the diagnosis of what many scent-sensitive individuals refer to as multiple chemical sensitivity, it creates room for unconventional medicine to fill the void, including homeopathy and naturopathy, says Dr. Susan Tarlo, a respiratory physician and professor of medicine at the University of Toronto in Ontario. "Unproven tests and unproven treatments have been offered to patients which is, in my opinion, very unfortunate," she says.

Given that a proportion of people are sensitive to scents, Tarlo views scentreduction policies as a good move - in some instances. "I think that's very reasonable for places like hospitals, even though we don't understand the mechanism, just to accommodate people who feel uncomfortable in that setting.'

Rather than implementing blanket bans on scented products, Dalton recommends that institutions address scent conflicts on a case-by-case basis. "As a general policy, it hasn't ever been shown to work very well," she says. "If somebody is wearing something that really bothers coworkers, I think it can be mitigated within the workplace."

Greg Noel, executive director of the Newfoundland and Labrador Lung Association, takes a similar approach in helping workplaces address scent sensitivities. "Most often, people will 
know what they are sensitive to and to what degree," Noel says. The solution could be as simple as moving an employee's desk to a new location. In one instance, an employee was sensitive to a floor cleaner and was able to recommend another cleaner that she could tolerate.

Noel also urges people to maintain an arm's-length policy when using scented products. If you can smell a perfume, body wash or deodorant from more than an arm's-length away, that person is wearing too much, he says.

Consumers should choose unscented or fragrance-free products, he adds. "Having no fragrance does not mean you are going to smell bad; it's not an issue of poor hygiene, or not using personal hygiene products."

When it comes to treating scentsensitive patients, Dalton advises that health care professionals take a measured approach and avoid alarmist messages that may increase anxiety. "Health care professionals themselves can over-sensitize some of their patients into believing they will have a reaction to fragrance material when, most likely, they won't." — Emily Senger, Toronto, Ont.

CMAJ 2011. DOI:10.1503/cmaj.109-3800 